\title{
Assessment of critical thresholds for cerebral perfusion pressure by performing bedside monitoring of cerebral energy metabolism
}

\author{
Carl-Henrik Nordström, M.D., Ph.D. \\ Department of Neurosurgery, Lund University Hospital, Lund Sweden
}

\begin{abstract}
An intractable increase in intracranial pressure (ICP) leading to a progressive decrease in cerebral perfusion pressure (CPP) and cerebral blood flow (CBF) is the dominating cause of death in patients with severe brain trauma. Arterial hypotension may further compromise CPP (and CBF) and significantly contributes to death. In addition, the injured brain is sensitive to raised CPP due to an increased permeability of the blood-brain barrier (BBB) to crystalloids and an impaired pressure autoregulation of the CBF. Given these circumstances, an increase in CPP will cause a net transport of water across the BBB and a further elevation in ICP. Accordingly, the assessment of the lower critical threshold for CPP is important for neurological intensive care. This level varies among different patients and different areas of the brain. In fact, the penumbral zones surrounding focal brain lesions appear to be the most sensitive. In the individual patient, preservation of normal cerebral energy metabolism within areas at risk during a decrease in CPP can be guaranteed by performing intracerebral microdialysis and bedside biochemical analyses.
\end{abstract}

KEY WORDS $\bullet$ blood-brain barrier $\bullet$ brain edema $\bullet$ brain trauma
cerebral perfusion pressure $\bullet$ intracranial pressure $\bullet$ microdialysis

An intractable increase in ICP leading to a progressive decrease in CPP and CBF is the dominant cause of death in patients with severe brain trauma. Arterial hypotension may further compromise CPP (and CBF), and hemorrhagic shock is a common complicating factor that significantly contributes to death. ${ }^{31}$ In addition, hypotensive episodes unrelated to hemorrhagic hypovolemia have been reported to be relatively frequent and associated with an unfavorable patient outcome. ${ }^{5}$ Furthermore, CBF is often variable in severe brain trauma, and regional CBF is frequently very low in pericontusional areas. ${ }^{15}$ Based on these experiences, it might seem logical both to restore circulating blood volume and to use vasopressors liberally to achieve a further increase in CPP. This idea has gained additional support from the (previously) generally accepted hypothesis that a pharmacologically induced increase in MABP effectively decreases ICP. ${ }^{35,36}$ In one study, Rosner, et al., ${ }^{36}$ asserted that the minimal level of CPP should be greater than $70 \mathrm{~mm} \mathrm{Hg}$ and that certain patients might require a level of $100 \mathrm{~mm} \mathrm{Hg}$ or more. Nonetheless, if the traumatized brain always benefits from increasing the CPP, what then is the point of assessing a minimal acceptable level? Perhaps an increased blood

\footnotetext{
Abbreviations used in this paper: $\mathrm{BBB}=$ blood-brain barrier; $\mathrm{CBF}=$ cerebral blood flow $\mathrm{CPP}=$ cerebral perfusion pressure; $\mathrm{CSF}=$ cerebrospinal fluid; GOS = Glasgow Outcome Scale; ICP = intracranial pressure; $\mathrm{MABP}=$ mean arterial blood pressure; $\mathrm{SD}=$ standard deviation.
}

pressure is harmful to the injured brain, which implies that many patients might benefit from a pharmacological reduction in CPP. In an effort to respond to these issues, I will first briefly review the regulation of brain volume under physiological and pathophysiological conditions.

\section{REGULATION OF BRAIN VOLUME}

All treatments for increased ICP are directed toward decreasing the volume of one or more of the following intracranial components (or, in selected cases, by increasing the total intracranial volume by craniectomy): volume of intracranial blood $\left(\mathrm{V}_{\text {blood }}\right)$, volume of brain tissue $\left(\mathrm{V}_{\text {brain }}\right)$, volume of intracranial CSF $\left(\mathrm{V}_{\mathrm{CSF}}\right)$, and volume of intracranial focal mass lesion $\left(\mathrm{V}_{\text {mass lesion }}\right)$. The relationship of these components is expressed in the following equation:

$$
\mathrm{V}_{\text {intracranial }}=\mathrm{V}_{\text {blood }}+\mathrm{V}_{\text {brain }}+\mathrm{V}_{\mathrm{CSF}}+\mathrm{V}_{\text {mass lesion }}
$$

Nonsurgical treatments are directed toward the volume of blood and the volume of brain tissue. A rapid but relatively short-lasting decrease in ICP is conventionally obtained by controlled hyperventilation therapy, which reduces the volume of intracranial blood. A more lasting decrease in intracranial blood volume may be achieved pharmacologically by precapillary vasoconstriction or by reducing blood volume within the venous compartment. ${ }^{12,27}$ The most effective, long-term nonsurgical treatment is to decrease the volume of the brain tissue, however. 
Volume regulation of all tissues is determined by the fluid exchange across the capillaries. Water transport across a microvascular bed $\left(\mathrm{J}_{\mathrm{v}}\right)$ is described in the following equation:

$\mathrm{J}_{\mathrm{v}}=\mathrm{L}_{\mathrm{p}} \times \mathrm{A} \times\left(\Delta \mathrm{P}-\Sigma \sigma_{\mathrm{s}} \times \Delta \Pi_{\mathrm{s}}\right)$

where $L_{p}$ represents the specific permeability for water (hydraulic conductivity) and A is the surface area available for fluid exchange. The product of $L_{p}$ and $A$ is denoted as the hydraulic conductance and reflects the total capacity for fluid exchange. Furthermore, $\Delta \mathrm{P}$ is the transcapillary hydrostatic pressure difference; $\Delta \pi_{\mathrm{s}}$, the transcapillary osmotic pressure difference; and $\sigma_{s}$, the reflection coefficient of each solute (s) of the system. Water exchange across the capillary wall is thus determined by the hydraulic conductance of the capillary wall $\left(\mathrm{L}_{\mathrm{p}} \times \mathrm{A}\right)$, the differences in hydrostatic pressure $(\Delta \mathrm{P})$ and osmotic pressure gradient $\left(\Delta \pi_{\mathrm{s}},\right)$ across the capillary wall, and the endothelial component determining which solutes are reflected and will contribute to the osmotic pressure gradient $\left(\sigma_{\mathrm{s}}\right)$. The value of $\sigma$ depends on the relative permeabilities of the membrane to water and to solute: if the membrane is impermeable to the solute but not to water, $\sigma$ equals 1 ; and if the permeability of the solute is identical to the diffusion coefficient in water, $\sigma$ equals 0 .

Volume control in the brain differs from that in other organs because of the BBB. In addition to its other vital functions, the $\mathrm{BBB}$ is the most important regulator of cerebral volume..$^{10}$ In other tissues, the osmotic force is mainly derived from the difference between plasma and interstitial colloid osmotic pressure, which approximately balance the transcapillary hydrostatic pressure $(\sim 20-25$ $\mathrm{mm} \mathrm{Hg}$ ). The situation across the BBB is quite different. Sodium and chloride, which are the two major solutes in biological fluids, have BBB reflection coefficients of 1 . Transcapillary fluid exchange across the BBB is thus effectively counteracted by the low permeability to crystalloids combined with their high osmotic pressure $(\sim 5700$ $\mathrm{mm} \mathrm{Hg}$ ) on both sides of the capillary wall. ${ }^{10}$ Water passing across the BBB in any direction will accordingly not be accompanied by crystalloids. If, due to a difference in hydrostatic pressure, water crosses the $\mathrm{BBB}$, an opposing osmotic gradient will immediately be created. Thus, brain volume operates within physiological conditions relatively independent of variations in intracapillary hydrostatic and colloid osmotic pressure. In addition, because of the pressure autoregulation of CBF, intracerebral capillary hydrostatic pressure functions within physiological conditions unaffected by variations in MABP. ${ }^{12,27}$

In patients with severe head injuries, autoregulation of CBF is often impaired, and variations in MABP will be directly reflected in the intracapillary hydrostatic pressure. ${ }^{7}$ Opinions vary regarding the permeability of the BBB in these patients. Data from experimental studies of brain trauma have shown that edema formation does not correspond with $\mathrm{BBB}$ opening and that this is not required for edema formation. ${ }^{3}$ In these studies only the permeability of large molecules was investigated, however, and it is reasonable to assume that given various pathological conditions, the BBB may have a significantly reduced reflection coefficient for crystalloids. As in other tissues, tran- scapillary fluid exchange will then be controlled according to the Starling equilibrium: water exchange across the capillary wall is determined by the differences in hydrostatic and colloid osmotic pressure between the intra- and extracapillary compartments. ${ }^{21}$ These pathophysiological conditions are probably valid in patients with severe brain trauma and should have a fundamental influence on neurological intensive care. According to these physiological principles, it is important not only to restore a normal circulating blood volume and a sufficient CPP, but also to normalize the colloidal osmotic pressure and to keep MABP (and hence intracapillary hydrostatic pressure) as low as possible. These physiological considerations constitute the basis for the volume-targeted therapy of high ICP (sometimes referred to as the "Lund concept"). ${ }^{1,9,12,27}$ Obviously, a reduction in MABP (and CPP) might jeopardize cerebral energy metabolism in these patients, especially in vulnerable regions such as the penumbral zones surrounding focal mass lesions. To eliminate these risks and to establish the critical thresholds for $\mathrm{CPP}$, patients with severe brain trauma are, in our intensive care unit, monitored with the aid of intracerebral microdialysis. Bedside analyses of variables reflecting cerebral energy metabolism have been conducted as a clinical routine since 1995.

\section{INTRACEREBRAL MICRODIALYSIS}

The technique of microdialysis was introduced more than 25 years ago for monitoring brains in animals ${ }^{43,45}$ and has become a standard procedure in neuroscience, with data from more than 8000 publications supporting its use. The first studies of the brain in humans were published more than 10 years ago. ${ }^{13,20}$ In 1995 CMA Microdialysis (Stockholm, Sweden) introduced microdialysis instruments for clinical use: catheters for peripheral and brain tissue, a microdialysis pump, and a bedside chemical analyzer. The microdialysis catheter designed for use in humans consists of a $60-\mathrm{mm}$-long shaft and a $10-\mathrm{mm}$-long dialysis membrane (polyamide, 20,000 molecular weight cutoff) with an outer diameter of $0.62 \mathrm{~mm}$ (CMA 70; CMA Microdialysis). The microdialysis catheters are perfused with a Ringer solution (perfusion fluid; CMA Microdialysis) from a microinfusion pump (CMA 106 or CMA 107; CMA Microdialysis) with a flow of 0.3 $\mu \mathrm{l} /$ minute during clinical routine monitoring. The microdialysis samples are collected in capped microvials (CMA Microdialysis) to prevent evaporation and are analyzed at bedside by using ordinary enzymatic methods on a microdialysis analyzer (CMA 600; CMA Microdialysis). The chemical variables of particular interest during intensive care are those related to glycolysis (glucose, pyruvate, and lactate), degradation of the glycerophospholipids of cell membranes (glycerol), and excessive levels of excitatory transmitters (glutamate). A simplified diagram of intermediary metabolism is featured in Fig. 1.

The measured levels of the chemical substances correspond to their absolute interstitial concentrations only if complete equilibration has occurred over the dialysis membrane. The degree of equilibration for a permeable substance (relative recovery) is mainly dependent on the perfusion rate, the length of the dialysis membrane, and 


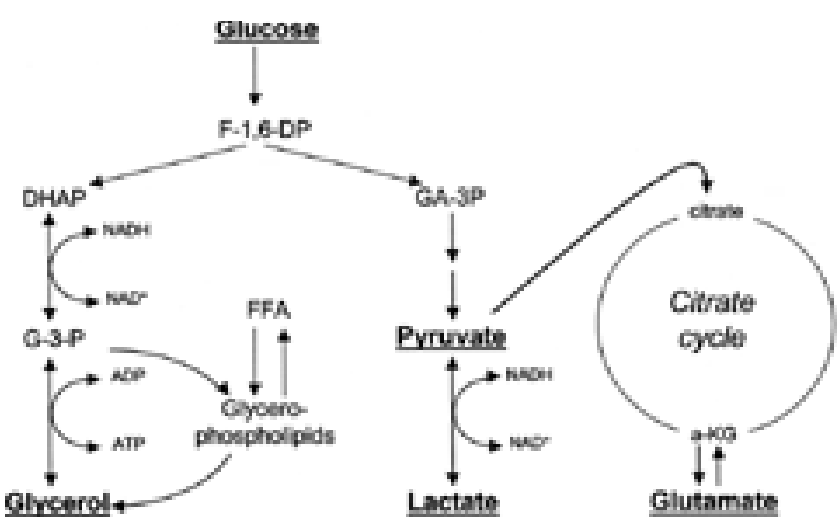

Fig. 1. Simplified diagram of intermediary metabolism of the glycolytic chain and its relation to the formation of glycerol and glycerophospholipids and to the citric acid cycle. $\alpha-\mathrm{KG}=\alpha$-ketoglutarate; DHAP = dihydroxyacetone-phosphate; F-1,6-DP = fructose-1,6-diposphate; FFA = free fatty acids; G-3-P = glycerol-3phosphate; GA-3P = glyceraldehyde-3-phosphate. Underlining indicates metabolites that are measured bedside by using enzymatic techniques.

the diffusion of the substance in the tissue. According to the clinical microdialysis equipment, the estimated recovery level for the substances mentioned earlier was approximately $70 \%$ of their true interstitial levels at a perfusion rate of $0.3 \mu \mathrm{l} /$ minute. ${ }^{16}$ Recovery may vary during pathophysiological conditions due to changed conditions of diffusion in the tissue. Note, however, that this variability is relevant mainly when performing studies of absolute concentration levels (for example, pharmacokinetic studies). ${ }^{8}$ During intensive care, the changes caused by the perturbation of energy metabolism are so profound that minor changes in relative recovery are of limited importance. Table 1 features data for these chemical variables in healthy, awake patients ${ }^{32}$ and in sedated patients with severe brain trauma and cerebral ischemia. ${ }^{40}$ The glycerol level and the lactate/pyruvate ratio are usually of particular interest during intensive care. A marked increase in the glycerol level indicates degradation of the glycerophospholipids of cell membranes. ${ }^{14,29,44}$ The lactate/pyruvate ratio reflects cytoplasmatic redox state, which can be expressed in terms of the lactate dehydrogenase equilibrium where $\mathrm{H}^{+}$stands for ionized hydrogen; NADH, the reduced form of nicotinamide-adenine dinucleotide; NAD ${ }^{+}$, the oxidized form of nicotinamide-adenine dinucleotide; and $\mathrm{K}_{\mathrm{LDH}}$ the equilibrium constant of the lactate dehydrogenase reaction: ${ }^{37}$
$[\mathrm{NADH}]\left[\mathrm{H}^{+}\right] /\left[\mathrm{NAD}^{+}\right]=[$lactate $] /[$pyruvate $] \times K_{\mathrm{LDH}}$

The time courses of the changes in these variables during transient cerebral ischemia are illustrated in Fig. 2. In this experimental study transient brain ischemia was induced in fetal lambs in utero by occluding the umbilical cord, followed by resuscitation after cardiac standstill (I Amer-Wahlin, et al., unpublished data). The microdialysis technique was identical to that used during clinical conditions, but the perfusion rate was increased $(1 \mu \mathrm{l} /$ minute) to allow frequent sampling. Induction of ischemia caused an almost instantaneous increase in the lactate/pyruvate ratio shortly thereafter, followed by an increase in the glutamate level. Glucose, pyruvate, and glutamate rapidly recovered after resuscitation, but the levels of lactate and glycerol remained elevated.

Use of the microdialysis technique provides biochemical information concerning only a small volume surrounding the catheter. This may be regarded as an advantage during neurological intensive care given that most adverse events primarily affect the sensitive penumbral zones surrounding focal lesions. ${ }^{41}$ Consequently, it is necessary to insert microdialysis catheters into these areas of interest. Figure $3 \mathrm{~A}$ and $\mathrm{B}$ illustrates the positions of two microdialysis catheters in areas close to superficial contusions following evacuation of an acute subdural hematoma. In addition, a third microdialysis catheter has been introduced close to the ventricular catheter in the opposite, less injured hemisphere (Fig. 3C).

\section{CEREBRAL ENERGY METABOLISM DURING A DECREASE IN CPP}

The lower critical limit of CPP for the maintenance of cerebral energy metabolism varies among different individuals (and different species). It also depends on the intra- and extracranial pathophysiological conditions (for example, whether the decrease in CPP was caused by an increase in ICP or a decrease in MABP (hypovolemia, cardiac failure, and so forth). A sudden increase in ICP in normal rats did not cause perturbation of cerebral energy metabolism until CPP had decreased to less than $30 \mathrm{~mm}$ Hg. ${ }^{38}$ An injured brain would be expected to be more sensitive. Furthermore, in a rat model of cranial impact injury, contusion volume was minimized when CPP was kept between 70 and $105 \mathrm{~mm} \mathrm{Hg}$ and increased at a CPP less than $60 \mathrm{~mm} \mathrm{Hg}$ or more than $105 \mathrm{~mm} \mathrm{Hg} .{ }^{19}$ These limits, which were obtained under experimental conditions, must not be directly transferred to human conditions, however.

In a consecutive series of 50 patients with severe trau-

TABLE 1

Cerebral interstitial concentrations of chemical variables during wakefulness in healthy patients and during ischemia in sedated patients with severe brain trauma*

\begin{tabular}{lcccccc}
\hline \hline \multicolumn{1}{c}{ Patient State } & $\begin{array}{c}\text { Glucose } \\
(\mathrm{mmol} / \mathrm{L})\end{array}$ & $\begin{array}{c}\text { Pyruvate } \\
(\mu \mathrm{mol} / \mathrm{L})\end{array}$ & $\begin{array}{c}\text { Lactate } \\
(\mathrm{mmol} / \mathrm{L})\end{array}$ & $\begin{array}{c}\text { Lactate/Pyruvate } \\
\text { Ratio }\end{array}$ & $\begin{array}{c}\text { Glutamate } \\
(\mu \mathrm{mol} / \mathrm{L})\end{array}$ & $\begin{array}{c}\text { Glycerol } \\
(\mu \mathrm{mol} / \mathrm{L})\end{array}$ \\
\hline healthy, awake & $1.7 \pm 0.9$ & $166 \pm 47$ & $2.9 \pm 0.9$ & $23 \pm 4$ & $16 \pm 16$ & $82 \pm 44$ \\
ischemic, sedated & $0.1 \pm 0.2$ & $31 \pm 47$ & $8.9 \pm 6.5$ & $460 \pm 560$ & $380 \pm 240$ & $570 \pm 430$ \\
\hline
\end{tabular}

*Data are given as the means \pm SD from Reinstrup, et al., and Ståhl, et al. ${ }^{40}$ 

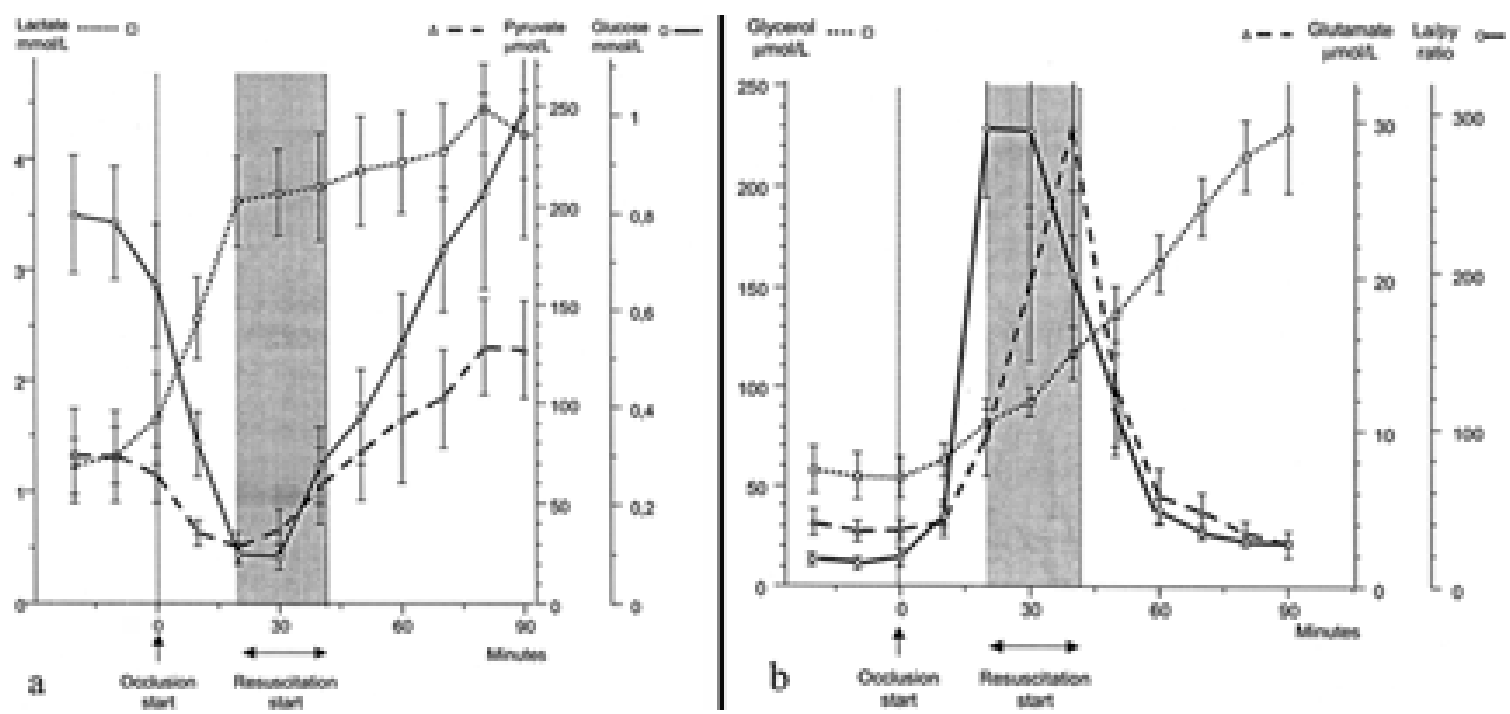

Fig. 2. Graphs demonstrating changes in intracerebral biochemistry in in utero fetal lambs after occlusion of the umbilical cord and resuscitation following cardiac standstill (I Amer-Wahlin, et al., unpublished data). a: Levels of glu-

matic brain injuries, multiple intracerebral microdialysis catheters were inserted to monitor energy metabolism while at a patient's bedside. ${ }^{28}$ All patients were treated surgically with the evacuation of focal, intracranial mass lesions. Thirty-one patients had an acute subdural hematoma (two were combined with an extradural hematoma and 15 were combined with focal cerebral contusions), three had a combination of focal contusion and extradural hematoma, 15 had pure focal hemorrhagic contusions, and one patient had a pure epidural hematoma. The microdialysis catheters were placed according to the principles in Fig. 3, that is, one or more catheters were positioned in the pericontusional penumbral zone (worse position) and one catheter was placed in the less injured hemisphere close to the ICP recording device (better posi- tion). All patients were treated according to the Lund concept $^{12,27}$ and the microdialysis samples were analyzed at bedside every 60 minutes.

Figure 4 shows the mean levels of glucose $(10,253$ measurements), lactate (11,538 measurements), and glycerol (13,244 measurements) and the lactate/pyruvate ratio (7704 measurements), from intracerebral microdialyses of the better and worse catheter positions in relation to four ranges of CPP. ${ }^{28}$ The interrupted horizontal lines indicate reference values (mean $\pm \mathrm{SD}$ ) for healthy brains in humans during wakefulness. ${ }^{32}$ As shown in Fig. 2, changes in glucose and the lactate/pyruvate ratio occurred instantaneously after the induction of brain ischemia, and the levels rapidly normalized after resuscitation. On the other hand, lactate and glycerol levels may remain in-
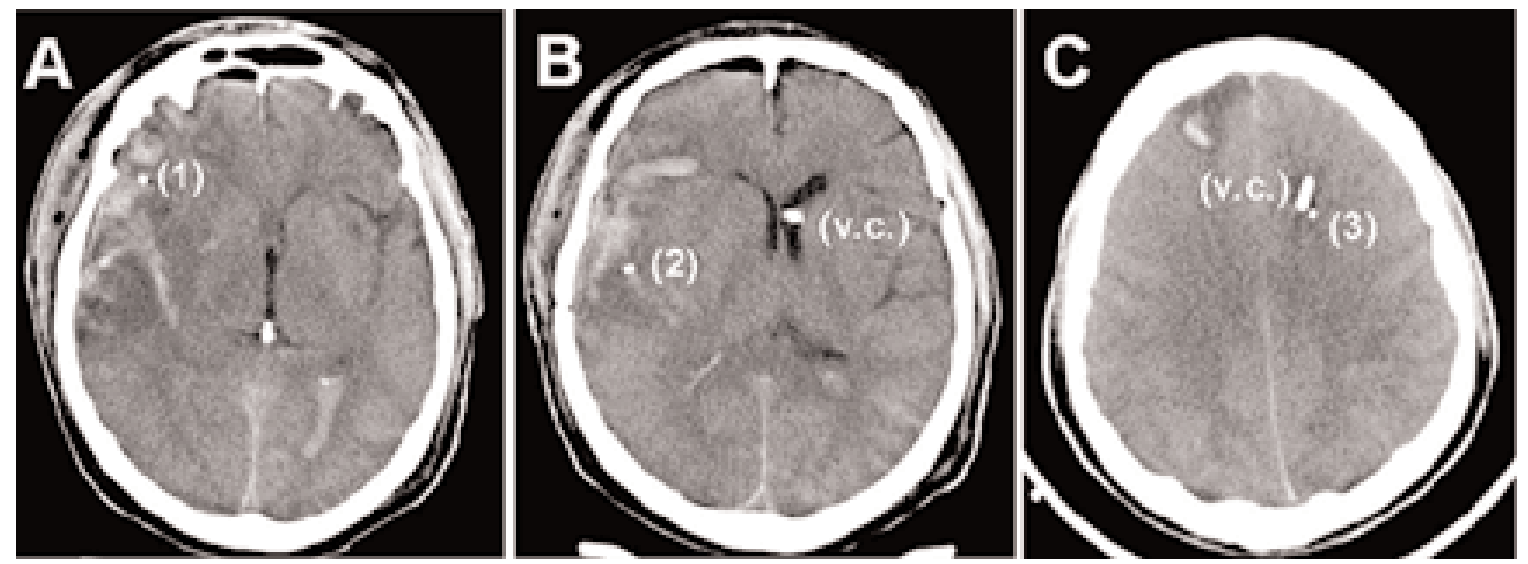

Fig. 3. Computerized tomography scans (A and B) demonstrating two microdialysis catheters (1 and 2) that have been positioned in areas close to superficial contusions following evacuation of an acute subdural hematoma. C: A computerized tomography scan demonstrating a third microdialysis catheter (3) that has been introduced close to the ventricular catheter (v.c.) in the opposite, less injured hemisphere. 

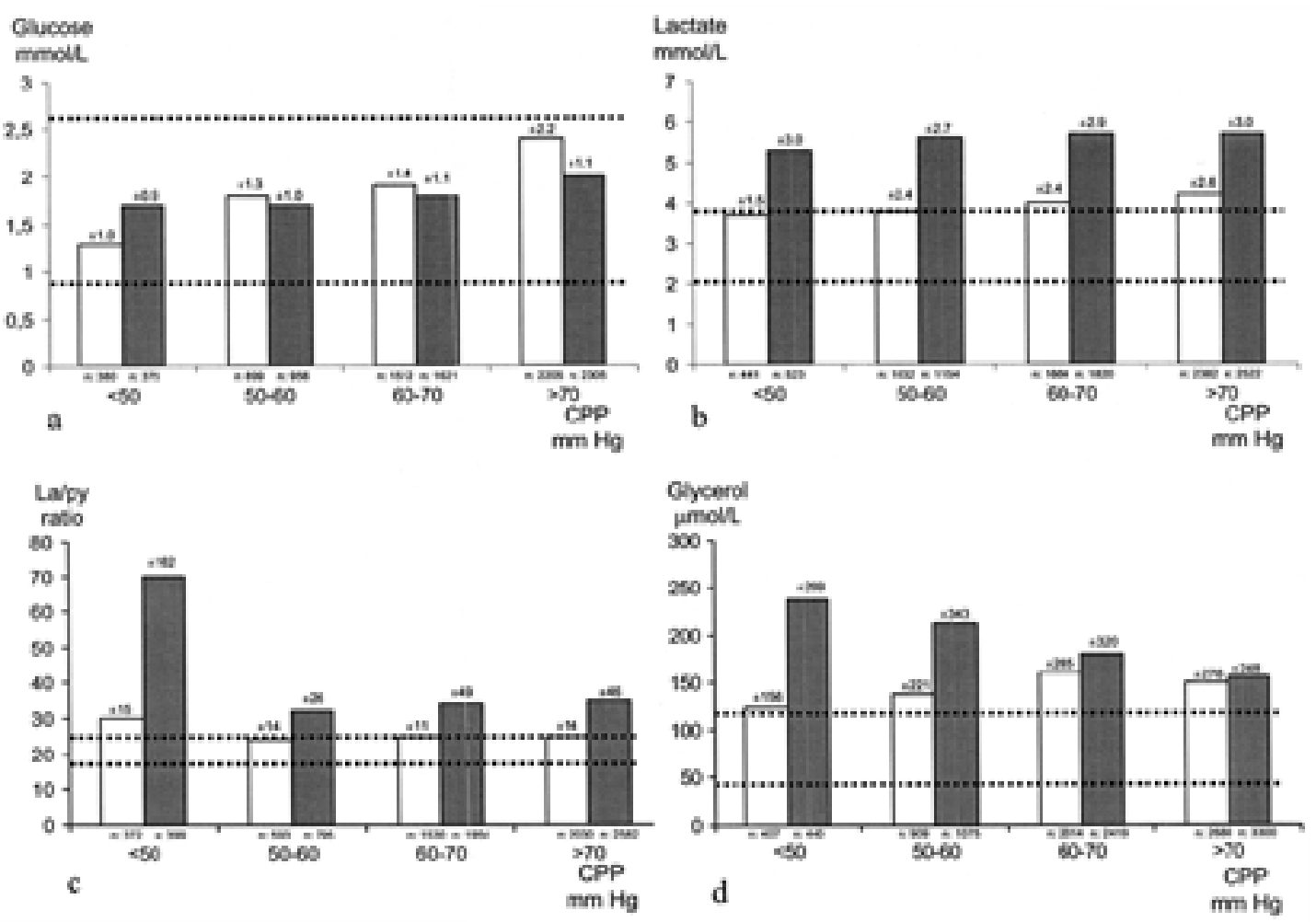

Fig. 4. Bar graphs demonstrating mean ( \pm SD) levels of glucose $(10,253$ measurements; a), lactate $(11,538$ measurements; b), lactate/pyruvate ratio (la/py) (7704 measurements; c), and glycerol (13,244 measurements; d) in the better (white bar) and worse (gray bar) catheter positions (see text) in relation to four ranges of CPPs in 50 patients with severe traumatic brain lesions. Interrupted lines indicate the range (mean $\pm \mathrm{SD}$ ) in healthy brains in humans during wakefulness.

creased for hours after transient ischemia (Fig. 2). Accordingly, we would expect the glucose level and the lactate/pyruvate ratio to be the most sensitive and specific indicators of the deterioration of cerebral energy metabolism during a transient decrease in CPP. In the present study, there were no statistically significant differences in glucose concentration when comparing the better and worse catheter positions or the different ranges of CPP. For the lactate/pyruvate ratio, the situation was more complex and a detailed statistical analysis is given in the original report. ${ }^{28}$ Briefly, no difference in lactate/pyruvate ratio was obtained when comparing the worse and better catheter positions at a CPP higher than $70 \mathrm{~mm} \mathrm{Hg}$. The lactate/pyruvate ratio was higher $(\mathrm{p}=0.04)$ in the worse than in the better catheter position at a CPP lower than 50 $\mathrm{mm} \mathrm{Hg}$. When comparing a CPP less than $50 \mathrm{~mm} \mathrm{Hg}$ with one greater than $70 \mathrm{~mm} \mathrm{Hg}$ and a CPP less than $50 \mathrm{~mm}$ $\mathrm{Hg}$ with one greater than $50 \mathrm{~mm} \mathrm{Hg}$, the lactate/pyruvate ratio was higher at the lower CPP in the worse catheter position ( $p=0.04$ and $p=0.01$, respectively). No difference in lactate/pyruvate ratio was obtained in the better catheter position when comparing a CPP less than $50 \mathrm{~mm}$ $\mathrm{Hg}$ to one greater than $50 \mathrm{~mm} \mathrm{Hg}$. These data support the view that, if necessary, CPP may be reduced to 50 to 60 $\mathrm{mm} \mathrm{Hg}$ in patients with severe traumatic brain lesions provided that the physiological and pharmacological principles followed in the study (Lund concept) are recognized.

The observation that the glucose concentration was within normal limits in the worse catheter position at CPP less than $50 \mathrm{~mm} \mathrm{Hg}$ (indicating a sufficient supply of the substrate [Fig. 4a]) even though the lactate/pyruvate ratio was very high (indicating an insufficient supply of oxygen [Fig. 4c]) is not contradictory. At a normal hemoglobin concentration of $150 \mathrm{~g} / \mathrm{L}$ and $95 \%$ saturation, human arterial blood contains approximately $9 \mu \mathrm{mol} \mathrm{O}_{2} / \mathrm{ml}$ and the arteriovenous $\mathrm{O}_{2}$ difference is approximately 3 $\mu \mathrm{mol} / \mathrm{ml} .^{28,37}$ Thus if CBF is reduced to approximately one third, theoretically all oxygen will be extracted. In practice, however, such a reduction in $\mathrm{CBF}$ cannot maintain a normal $\mathrm{O}_{2}$ supply, because the reduction in $\mathrm{PO}_{2}$ will also decrease the $\mathrm{O}_{2}$ diffusion gradient. For glucose, the following approximate levels have been described in humans: arterial concentration $5.1 \mu \mathrm{mol} / \mathrm{ml}$, venous concentration $4.6 \mu \mathrm{mol} / \mathrm{ml}$, arteriovenous glucose difference $0.5 \mu \mathrm{mol} / \mathrm{ml} .{ }^{28,37}$ Accordingly, during ischemia induced by a gradual decrease in $\mathrm{CBF}, \mathrm{O}_{2}$ supply to the brain will be insufficient before the supply of substrate is seriously jeopardized.

\section{BIOCHEMICAL EVALUATION OF TISSUE OUTCOME}

The efficacy of any therapy administered during neurological intensive care is conventionally judged based on clinical outcome. The microdialysis technique offers an additional possibility: restitution of energy metabolism 
and intermediary metabolism may be used as an indicator of tissue outcome. It is then important to evaluate the biochemical features not only in less injured parts of the brain, but also in the more vulnerable penumbral zones (Fig. 3).

Figure 5 summarizes physiological and biochemical information in a series of patients treated according to the Lund concept. ${ }^{42}$ The figure graphically shows changes in MABP and CPP (Fig. 5a) as well as intracerebral glycerol levels (Fig. 5b) during the first 72 hours after the start of treatment. In accordance with the Lund concept, CPP decreased from 73 to $62 \mathrm{~mm} \mathrm{Hg}$ due to the pharmacologically induced reduction in MABP. The gradual decrease in ICP caused a slow increase in CPP back to 65 to $70 \mathrm{~mm}$ $\mathrm{Hg}$ following the initial decrease. The pharmacologically induced decrease in CPP was associated with a simultaneous decrease in interstitial glycerol in the worse catheter position from a very high level $(>300 \mu \mathrm{mol} / \mathrm{L})$ at the start of treatment to a normal level within 72 hours. These data strongly support the view that the vulnerable penumbral zone may tolerate a controlled decrease in CPP provided that this is achieved according to the physiological principles discussed earlier.

\section{CLINICAL ASPECTS}

For two reasons many authors have regarded a substantial increase in CPP, often accomplished by the infusion of vasopressors, as desirable in patients with severe brain trauma. First, the injured brain is exceptionally vulnerable to secondary ischemic insults (in particular, hypotension), which are associated with a poor neurological outcome. ${ }^{6,22}$ Accordingly, it might seem rational to use vasopressors liberally to increase CPP. In a randomized clinical trial, $\mathrm{CPP}$ was maintained above $70 \mathrm{~mm} \mathrm{Hg}$ to improve CBF and prevent secondary ischemic insults..$^{34}$ The use of this CBF-targeted therapy successfully reduced the incidence and duration of jugular venous desaturation; however, it also increased the incidence of adult respiratory distress syndrome and failed to improve neurological outcome. ${ }^{34}$ Consequently, the study data did not support the liberal use of vasopressors, and it was later concluded that a CPP of $60 \mathrm{~mm} \mathrm{Hg}$ probably provided adequate perfusion for most patients with severe traumatic brain injuries. ${ }^{33}$

Second, it has been asserted that a decrease in CPP provokes an increase in ICP (vasodilatory cascade) and, conversely, that an increase in CPP effectively decreases ICP (vasoconstriction cascade). ${ }^{35,36}$ The notion that an increase in CPP would regularly cause a decrease in ICP receives little support from physiological study data. In an experimental study of the injured feline brain, induced arterial hypertension invariably increased ICP. ${ }^{18}$ Furthermore, in a recent clinical study of patients with severe brain trauma a titratable phenylephrine infusion used to increase in MABP by 10 to $15 \mathrm{~mm} \mathrm{Hg}$ significantly increased ICP in almost all studied cases. ${ }^{30}$

The earlier theoretical physiological discussion strongly supports the view that the injured brain would benefit from maintaining a low intracapillary hydrostatic pressure and a normal colloid osmotic pressure. Data from the clinical microdialysis studies presented earlier document that the injured brain tolerates a controlled decrease in CPP according to the Lund concept. This therapy is focused on a pharmacologically induced reduction in MABP to reduce hydrostatic capillary pressure, colloid infusion to increase plasma oncotic pressure, and antistress therapy and maintenance of a normal circulating blood volume to improve microcirculation..$^{1,9,12,27}$ A reduction of the intracranial blood volume is achieved by reducing cerebral energy metabolism, and a further reduction in blood volume, if

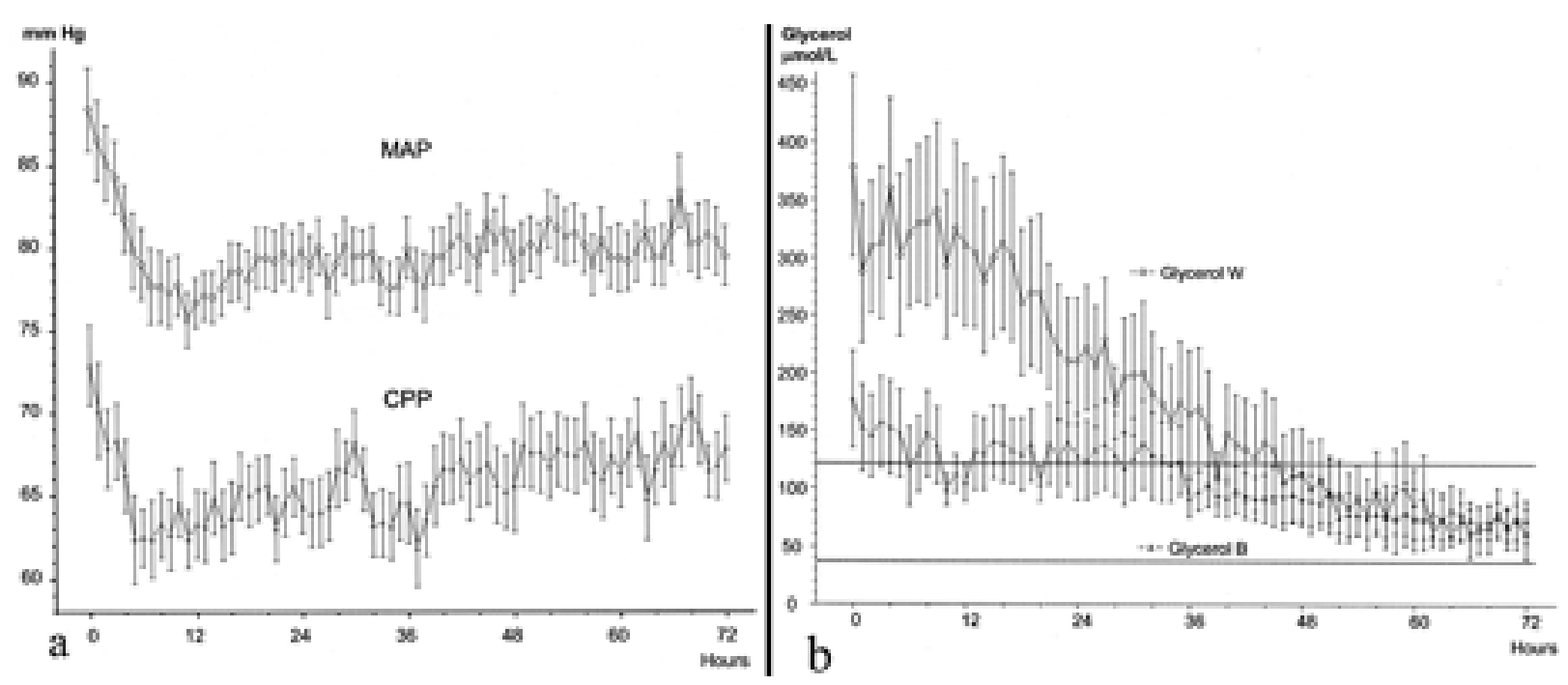

Fig. 5. a: Graph demonstrating the reductions in MABP and CPP during the first 72 hours after the start of treatment according to the Lund concept (mean \pm standard error of the mean). b: Graph demonstrating glycerol levels (mean \pm standard error of the mean) obtained bedside from microdialysis catheters postioned in the penumbral zone surrounding evacuated focal brain lesions (Glycerol W) as well as from the contralateral hemisphere (Glycerol B). Interrupted lines indicate the range (mean \pm SD) in healthy brains in humans during wakefulness. The intentional decrease in CPP was associated with a normalization of all studied biochemical variables. 
Cerebral perfusion pressure and brain energy metabolism

TABLE 2

Outcome according to the GOS in patients with severe traumatic brain lesions treated conventionally and according to the Lund concept*

\begin{tabular}{|c|c|c|c|c|c|c|c|c|}
\hline \multirow[b]{2}{*}{ Authors \& Year } & \multirow[b]{2}{*}{ Study Years } & \multirow[b]{2}{*}{ No. of Patients } & \multirow{2}{*}{$\begin{array}{l}\text { Treatment } \\
\text { Protocol }\end{array}$} & \multicolumn{5}{|c|}{ GOS Score ( $\%$ of patients) } \\
\hline & & & & GR & MD & SD & VS & $\mathrm{D}$ \\
\hline Eker, et al., 1998 & $1982-1986$ & 38 & $\begin{array}{l}\text { hyperventilation } \\
\& \text { high-dose } \\
\text { barbituate coma }\end{array}$ & 29 & 13 & 5 & 5 & 47 \\
\hline Eker, et al., 1998 & 1989-1994 & 53 & Lund concept & 38 & 42 & 11 & 2 & 8 \\
\hline Naredi, et al., 1998 & 1993-1994 & 38 & Lund concept & 42 & 29 & 13 & 3 & 11 \\
\hline Naredi, et al., 2001 & 1998 & 31 & Lund concept & 61 & 10 & 23 & 3 & 3 \\
\hline
\end{tabular}

* $\mathrm{D}$ = death; $\mathrm{GR}=$ good recovery; $\mathrm{MD}=$ moderate disability; $\mathrm{SD}=$ severe disability; $\mathrm{VS}$ = vegetative state.

necessary, can be obtained within the venous compartment by infusing dihydroergotamine..$^{2,25,26}$ The goal of this treatment is to reach a slow and lasting reduction in ICP over hours to days. Detailed accounts of the drugs and dosages used in this treatment protocol have been presented in previous reports. ${ }^{1,9,12,27}$

Results of randomized controlled studies are used definitively to prove the efficacy of all treatment protocols, and the Lund concept has been criticized for not being proven in this way. ${ }^{11}$ Currently, there are no such studies to support any specific treatment for increased ICP, however. ${ }^{4,39}$ Until data from such studies are available, the efficacy of the Lund concept may be judged based on the physiological and biochemical studies discussed earlier, and the clinical results compared with historical controls. Clinical outcome following the use of the Lund concept has been reported by investigators from three neurosurgical centers in Sweden. ${ }^{9,23,24}$ The clinical results 6 months after injury, according to the GOS, ${ }^{17}$ are listed in Table 2. In the original study, in which were compared two groups of patients with very severe brain trauma, impaired $\mathrm{CO}_{2}$ reactivity, and an ICP above $25 \mathrm{~mm} \mathrm{Hg}$ despite conventional treatment, the mortality rate decreased from 47 to $8 \%$. $^{9}$ Significant increases in the groups with a good recovery and moderate disability were obtained, but there was no increase in the number of patients in the groups with severe disability or vegetative state.

\section{CONCLUSIONS}

The injured brain is exceptionally vulnerable to secondary ischemic insults (for example, arterial hypotension), but also-due to an increased permeability of the $\mathrm{BBB}$ to crystalloids and an impaired pressure autoregulation of $\mathrm{CBF}$ - sensitive to increased MABP. An increase in CPP (and a decrease of the colloid osmotic pressure of the blood) will, given these circumstances, lead to a net transport of water across the BBB and an increase in ICP. Accordingly, an assessment of the lower critical threshold for CPP is important for neurological intensive care. This threshold varies among different patients and different areas of the brain, and the penumbral zones surrounding focal brain lesions appear to be the most sensitive. ${ }^{15,33,41}$ Thus, the optimal treatment protocol for patients with severe traumatic brain lesions and increased ICP probably differs among patients. In an individual patient, the preservation of normal cerebral energy metabolism within areas at risk during a decrease in CPP can be guaranteed by performing intracerebral microdialysis and bedside biochemical analyses. ${ }^{28}$

\section{References}

1. Asgeirsson B, Grände PO, Nordström $\mathrm{CH}$ : A new therapy of post-trauma brain oedema based on haemodynamic principles for brain volume regulation. Intensive Care Med 20:260-267, 1994

2. Asgeirsson B, Grände PO, Nordström CH, et al: Cerebral haemodynamic effects of dihydroergotamine in patients with severe traumatic brain lesions. Acta Anaesthesiol Scand 39: 922-930, 1995

3. Beaumont A, Marmarou A, Hayasaki K, et al: The permissive nature of blood brain barrier (BBB) opening in edema formation following traumatic brain injury. Acta Neurochir Suppl 76:125-129, 2000

4. Brain Trauma Foundation: Guidelines for Surgical Management of Traumatic Brain Injury. (http://www2.braintrauma.org/guidelines) [Accessed 12 November 2003]

5. Chesnut RM, Gautille T, Blunt BA, et al: Neurogenic hypotension in patients with severe head injuries. J Trauma 44: 958-964, 1998

6. Chesnut RM, Marshall LF, Klauber MR, et al: The role of secondary brain injury in determining outcome from severe head injury. J Trauma 34:216-222, 1993

7. Cold GE, Jensen FT: Cerebral autoregulation in unconscious patients with brain injury. Acta Anaesthesiol Scand 22: 270-280, 1978

8. Ederoth P, Tunblad K, Bouw R, et al: Blood-brain barrier transport of morphine in patients with severe brain trauma. $\mathbf{B r} \mathbf{J}$ Clin Pharmacol (In press)

9. Eker C, Asgeirsson B, Grände PO, et al: Improved outcome after severe head injury with a new therapy based on principles for brain volume regulation and preserved microcirculation. Crit Care Med 26:1881-1886, 1998

10. Fenstermacher JD: Volume regulation of the central nervous system, in Staub NC, Taylor AE (eds): Edema. New York: Raven Press, 1984, pp 383-404

11. Gisvold SE: The Lund concept for treatment of head injuriesfaith or science? Acta Anaesthesiol Scand 45:399-401, 2001

12. Grände PO, Asgeirsson B, Nordström $\mathrm{CH}$ : Volume-targeted therapy of increased intracranial pressure: the Lund concept unifies surgical and non-surgical treatments. Acta Anaesthesiol Scand 46:929-941, 2002

13. Hillered L, Persson L, Pontén U, et al: Neurometabolic monitoring of the ischaemic human brain using microdialysis. Acta Neurochir 102:91-97, 1990

14. Hillered L, Valtysson J, Enblad P, et al: Interstitial glycerol as a 
marker for membrane phospholipid degradation in the acutely injured human brain. J Neurol Neurosurg Psychiatry 64: 486-491, 1998

15. Hlatky R, Valadka AB, Robertson CS: Intracranial hypertension and cerebral ischemia after severe traumatic brain injury. Neurosurg Focus 14 (4): Article 2, 2003

16. Hutchinson PJ, O'Connell MT, al-Rawi PG, et al: Clinical cerebral microdialysis - determining the true extracellular concentration. Acta Neurochir Suppl 81:359-362, 2002

17. Jennett B, Bond M: Assessment of outcome after severe brain damage. Lancet 1:480-484, 1975

18. Kongstad L, Grände PO: Arterial hypertension increases intracranial pressure in cat after opening of the blood-brain barrier. J Trauma 51:490-496, 2001

19. Kroppenstedt SN, Kern M, Thomale UW, et al: Effect of cerebral perfusion pressure on contusion volume following impact injury. J Neurosurg 90:520-526, 1999

20. Meyerson BA, Linderoth B, Karlsson H, et al: Microdialysis in the human brain: extracellular measurements in the thalamus of parkinsonian patients. Life Sci 46:301-308, 1990

21. Michel CC: Starling: the formulation of his hypothesis of microvascular fluid exchange and its significance after 100 years. Exp Physiol 82:1-30, 1997

22. Miller JD, Sweet RC, Narayan RK, et al: Early insults to the injured brain. JAMA 240:439-442, 1978

23. Naredi S, Edén E, Zäll S, et al: A standardized neurosurgical neurointensive therapy directed toward vasogenic edema after severe traumatic brain injury: clinical results. Intensive Care Med 24:446-451, 1998

24. Naredi S, Olivercrona M, Lindgren C, et al: An outcome study of severe traumatic head injury using the "Lund therapy" with low-dose prostacyclin. Acta Anaesthesiol Scand 45:402-406, 2001

25. Nilsson F, Messeter K, Grände PO, et al: Effects of dihydroergotamine on cerebral circulation during experimental intracranial hypertension. Acta Anaesthesiol Scand 39:916-921, 1995

26. Nilsson F, Nilsson T, Edvinsson L, et al: Effects of dihydroergotamine and sumatriptan on isolated human cerebral and peripheral arteries and veins. Acta Anaesthesiol Scand 41: 1257-1262, 1997

27. Nordström CH: Treatment of increased intracranial pressure: physiological and biochemical principles underlying volume targeted therapy-the "Lund concept." Neurocrit Care (In press)

28. Nordström $\mathrm{CH}$, Reinstrup $\mathrm{P}, \mathrm{Xu} \mathrm{W}$, et al: Assessment of the lower limit for cerebral perfusion pressure in severe head injuries by bedside monitoring of regional energy metabolism. Anesthesiology 98:809-814, 2003

29. Nordström $\mathrm{CH}$, Ungerstedt U: Intracerebral microdialysis with bedside analysis of glucose, lactate, glycerol and urea, in Diemath HE, Sommerauer J, von Wild KRH (eds): Brain Protection in Severe Head Injury. New York: W Zuckschwerdt Verlag, 1996, pp 117-119

30. Oertel M, Kelly DF, Lee JH, et al: Efficacy of hyperventilation, blood pressure elevation, and metabolic suppression therapy in controlling intracranial pressure after head injury. J Neurosurg 97:1045-1053, 2002
31. Piek J, Chesnut RM, Marshall LF, et al: Extracranial complications of severe head injury. J Neurosurg 77:901-907, 1992

32. Reinstrup P, Ståhl N, Mellergard P, et al: Intracerebral microdialysis in clinical practice: baseline values for chemical markers during wakefulness, anesthesia, and neurosurgery. Neurosurgery 47:701-710, 2000

33. Robertson CS: Management of cerebral perfusion pressure after traumatic brain injury. Anesthesiology 95:1513-1517, 2001

34. Robertson CS, Valadka AB, Hannay HJ, et al: Prevention of secondary ischemic insults after severe head injury. Crit Care Med 27:2086-2095, 1999

35. Rosner MJ, Becker DP: Origin and evolution of plateau waves. Experimental observations and a theoretical model. J Neurosurg 60:312-324, 1984

36. Rosner MJ, Rosner SD, Johnson AH: Cerebral perfusion pressure: management protocol and clinical results. J Neurosurg 83:949-962, 1995

37. Siesjö BK: Brain Energy Metabolism. New York: Wiley, 1978

38. Siesjö BK, Zwetnow NN: Effects of increased cerebrospinal fluid pressure upon adenine nucleotides and upon lactate and pyruvate in rat brain tissue. Acta Neurol Scand 46:187-202, 1970

39. Slavik RS, Rhoney DH: Pharmacological management of severe traumatic brain injury: an evidence-based review. (http://www.informedpharmacotherapy.com/Issue3/IPP/Slavik TBI.htm) [Accessed 12 November 2003]

40. Ståhl N, Mellergård P, Hallström Å, et al: Intracerebral microdialysis and bedside biochemical analysis in patients with fatal traumatic brain lesions. Acta Anaesthesiol Scand 45:977-985, 2001

41. Ståhl N, Schalén W, Ungerstedt U, et al: Bedside biochemical monitoring of the penumbra zone surrounding an evacuated acute subdural haematoma. Acta Neurol Scand 108:211-215, 2003

42. Ståhl N, Ungerstedt U, Nordström CH: Brain energy metabolism during controlled reduction of cerebral perfusion pressure in severe head injuries. Intensive Care Med 27:1215-1223, 2001

43. Ungerstedt U: Microdialysis—-principles and applications for studies in animal and man. J Intern Med 230:365-373, 1991

44. Ungerstedt U, Bäckström T, Hallström $\AA$, et al: Microdialysis in normal and injured human brain, in Kinney JM, Tucker HN (eds): Physiology, Stress, and Malnutrition: Functional Correlates, Nutritional Intervention. Philadelphia: Lippincott-Raven, pp 361-381, 1997

45. Ungerstedt U, Pycock CH: Functional correlates of dopamine neurotransmission. Bull Schweiz Acad Med Wiss 30:44-55, 1974

Manuscript received October 21, 2003.

Accepted in final form November 12, 2003.

Address reprint requests to: Carl-Henrik Nordström, M.D., Ph.D., Department of Neurosurgery, Lund University Hospital, S-22185 Lund, Sweden. email: carl-henrik.nordstrom@neurokir.lu.se. 